\title{
Moving in the Right Direction - Articles From Ontario's Systems Enhancement Evaluation Initiative (SEEI)
}

\author{
Lindsey George and Janet Durbin
}

Welcome to this special edition of the Canadian Journal of Community Mental Health. We are delighted to be guest editors of this issue and to be given the privilege to assist in presenting the hard work of many outstanding researchers committed to bringing new understandings to the community mental health sector. We gratefully acknowledge the instrumental role of Dr. Paula Goering in creating this unique opportunity for researchers and other mental health system stakeholders in Ontario.

Beginning in 2005, Ontario's Ministry of Health and Long-Term Care made a 52\% increase in investments to the community mental health sector. A total of $\$ 167$ million was infused into community mental health services in the province. This enhancement included increases in funding for crisis services, intensive case management, court diversion and support, assertive community treatment, and early intervention in psychosis. The Ministry also committed dollars to evaluating the impact of these investments. The result of that investment in evaluation is contained in these pages. The researchers presenting their findings are all dedicated to the ongoing challenge of figuring out what is working and what we can do better. This was the goal of the Systems Enhancement Evaluation Initiative (SEEI).

The Health Systems Research and Consulting Unit, Centre for Addiction and Mental Health, directed by Dr. Goering, led the Systems Enhancement Evaluation Initiative. The projects brought together researchers and stakeholders to develop and undertake system evaluation research to better understand the impact of increased investments in community mental health. After a peer-reviewed process facilitated by the Ontario Mental Health Foundation, nine studies were approved. The studies cover a wide range of approaches including system-level and site-specific evaluation projects, and use a variety of research methods. The results are rich in detailing the complexity of program development and implementation in mental health care.

What is most exciting for us is the unique opportunity to tackle messy, real-world evaluation researchmuch like the messy, real world people find themselves in when they seek help for mental health problems. Our research world is not about undertaking the perfectly designed research study. It is about joining with persons living with mental illness, families, providers, decision-makers and other researchers to try to figure out what is working and what we can improve on. The research studies presented here involved investigators from a variety of backgrounds in the work. These individuals helped us to develop the research questions 
and methods, interpret the data, and make recommendations based on the results. What readers find in these pages is the work of many people, not simply those whose names are on the papers. We wish to thank everyone who contributed to producing these results. It is not easy to undertake this kind of research, but it is quite exhilarating when we learn more about what we do.

Program evaluation research is, by its nature, messy. It is also critical to moving us forward in understanding how to improve models and systems of care.

Some of the challenges we faced

- Data are imperfect.

- Involving other stakeholders takes commitment and time.

- When money flows, it is not always timed for good evaluation.

- The impact of new investments may not be realized for several years, particularly as new or enhanced program changes take considerable time to implement.

\section{What we learned from the studies}

- New investments in community mental health have made an impact on those seeking mental health services.

- $\quad$ People using mental health services and their families are, on balance, very positive about the services available to them.

- Systems of care are complex. Infusions of new funds may make a difference in ways that are unanticipated. Increased resources, with attendant increase in awareness of them, may create an increased demand for services rather than a reduction on service demands. More people accessing the system may be the success story.

- Effectively measuring impact requires much better data systems across jurisdictions. People living with mental health issues are served by a variety of government and non-governmental programs. To measure the impact of any given program, we must account for what occurs in other sectors-a feat that is currently beyond our measurement ability.

- Data systems must be based on individual data, to enable tracking of people across time and service use, including those who leave the care systems, in order to accurately assess the impact of investments in community mental health.

- Doing messy research is fun and gratifying. It makes us accountable to our community and provides valuable insights into our mental health system, with all of its imperfections.

The 11 papers in this special issue present a range of findings from the funded studies. The issue starts with an analysis of the process of gaining meaningful involvement of stakeholders in this research endeavour to increase the relevance of findings. It is followed by evaluations of provincial or local area responses to the enhancements. The final set of studies assess the experience of specific organizations. One study, the Waterloo-Wellington Crisis System Evaluation, is not represented in these papers, but a detailed report of the study and its results can be found at www.ehealthontario.ca. Waterloo-Wellington used the new funding to expand crisis services and improve crisis system coordination, in part through creation of a crisis system 
coordinator position. Findings show a system in change, with many positive results and some continuing challenges.

We look forward to continued efforts in Ontario to develop our systems of care for persons with mental health issues, and to evaluate these developments through collaborative research with the rich diversity of individuals who want to make our systems better. 